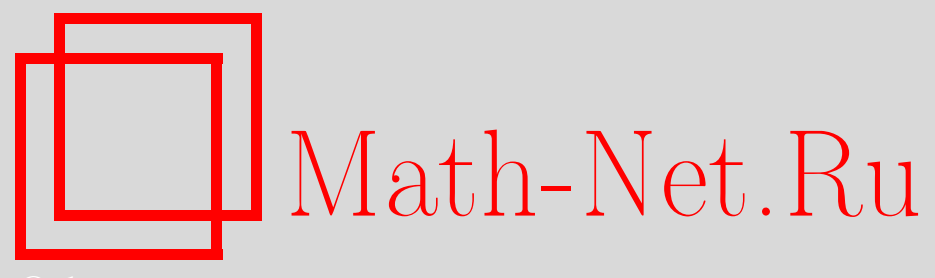

Д. В. Шуваев, Вычисление распределения одной комбинаторной статистики, заданной на последовательностях с фиксированным составом знаков, Матем. вопр. криптогр., 2011, том 2, выпуск 3, 99-109

DOI: https://doi.org/10.4213/mvk38

Использование Общероссийского математического портала Math-Net.Ru подразумевает, что вы прочитали и согласны с пользовательским соглашением http://www . mathnet.ru/rus/agreement

Параметры загрузки:

IP : 35.174 .16 .151

26 апреля 2023 г., 11:15:16 


\title{
Вычисление распределения одной комбинаторной статистики, заданной на последовательностях с фиксированным составом Знаков
}

\author{
Д. В. Шуваев \\ Лаборатория ТВП, Москва
}

Получено 10.V.2010

Для конечной целочисленной последовательности $\alpha$ рассматривается число $X(\alpha)$ чтений $\alpha$ слева направо, необходимое для обращения ко всем элементам последовательности в порядке неубывания. Получены формулы для вычисления точного распределения $X(\alpha)$, когда $\alpha$ равновероятно распределена на множестве всех перестановок $n$ заданных чисел.

Ключевые слова: число чтений, цепь Маркова

Computing the distribution of a combinatorial statistics defined on permutations of a given multiset

\section{V. Shuvaev}

\section{TVP Laboratory, Moscow}

\begin{abstract}
For a finite integer sequence $\alpha$ we consider the number $X(\alpha)$ of readings of $\alpha$ from left to right necessary to count all elements of the sequence in a nondecreasing order. We describe a method to calculate the distribution of $X(\alpha)$ exactly when $\alpha$ is uniformly distributed on the set of all permutations of $n$ given numbers.
\end{abstract}

Key words: number of readings, Markov chain

Citation: Mathematical Aspects of Cryptography, 2011, vol. 2, no. 3, pp. 99-109 (Russian). 
Говорят, что конечная числовая последовательность имеет $s$ чтений, если ее нужно просмотреть $s$ раз слева направо, чтобы в порядке неубывания вычеркнуть все ее элементы. Элементы, вычеркиваемые в ходе одного просмотра слева направо, образуют одно чтение. Таким образом, вся последовательность разбивается на чтения. Ясно, что совпадающие элементы последовательности принадлежат одному чтению. Вместе с тем чтение может содержать несовпадающие элементы. Пример: последовательность 3, 5, 3, 4, 1, 5, 2 содержит три чтения - $(1,2),(3,3,4),(5,5)$.

Вопрос о распределении числа чтений в случайной последовательности связан, в частности, с задачами о сортировке, см., например [3]. Наиболее полно исследовано распределение числа чтений в случайной равновероятной перестановке порядка $n$. Нетрудно проверить, что число чтений перестановки $\alpha=\left(\alpha_{1}, \alpha_{2}, \ldots, \alpha_{n}\right)$ на единицу превосходит число убываний в обратной перестановке, т. е. число выполненных соотношений в следующем ряду: $\left(\beta_{1}>\beta_{2}\right),\left(\beta_{2}>\beta_{3}\right), \ldots,\left(\beta_{n-1}>\beta_{n}\right)$, где $\beta=\left(\beta_{1}, \beta_{2}, \ldots, \beta_{n}\right)=\alpha^{-1}$. Имея в виду, что убывания разбивают последовательность (перестановку) на отрезки неубывания (отрезки возрастания), можно сказать, что число чтений перестановки равно числу отрезков возрастания в обратной перестановке.

Перечисление перестановок по числу убываний описывается так называемыми числами Эйлера $A_{n, k}, n \geqslant 1,1 \leqslant k \leqslant n\left[6\right.$, с. 55]. Именно, число $A_{n, k}$ равно числу перестановок степени $n$, имеющих ровно $k-1$ убывание. Числа Эйлера можно определить следующим способом:

$$
\begin{gathered}
A_{1, k}=\chi\{k=1\}, \\
A_{n, k}=k A_{n-1, k}+(n-k+1) A_{n-1, k-1} \quad(n \geqslant 2,1 \leqslant k \leqslant n),
\end{gathered}
$$

здесь и далее $\chi$ - индикатор события, стоящего в скобках: $\chi\{B\}=1$, если $B$ истинно, $\chi\{B\}=0$, если $B$ ложно.

Указанную формулу, а также другие способы задания чисел Эйлера, можно найти в книгах [3-5]. Асимптотические свойства чисел Эйлера описаны в $[5,17]$. Для случайной равновероятной перестановки известны совместные распределения числа чтений и числа убываний, числа чтений и числа инверсий, числа чтений и числа инверсий в обратной перестановке, числа чтений и так называемого мажорирующего индекса перестановки $[9,16]$.

Общий случай, когда случайная последовательность может содержать совпадающие элементы, несколько сложнее. Перечисление последовательностей с заданным составом знаков по числу убываний составляет так называемую задачу С. Ньюкомба, ее решение можно найти в $[4,8]$. В отличие от случайных перестановок, для случайных последовательностей распределение 
числа отрезков невозрастания и распределение числа чтений могут не совпадать.

В работах $[1,2]$ исследованы асимптотические свойства распределений числа чтений для некоторых классов случайных последовательностей, в том числе для последовательностей с заданным составом знаков. Настоящая работа посвящена задаче точного вычисления распределения числа чтений в случайной последовательности с фиксированным составом знаков.

Пусть $1<m \leqslant n, M=\{1,2, \ldots, m\}$. Таким образом, $M^{n}-$ это множество последовательностей длины $n$, составленных из элементов множества $M$.

Обозначим через $S[s]$ подмножество $M^{n}$, состоящее из последовательностей со спецификацией $[s]=\left[s_{1}, s_{2}, \ldots, s_{m}\right]$, т. е. состоящих из $s_{1}$ единиц, $s_{2}$ двоек, $\ldots, s_{m}$ чисел $m$, где $n=s_{1}+\cdots+s_{m}$. Обозначим $X(\alpha)$ число чтений в последовательности $\alpha$. Всюду далее считаем, что последовательность $\alpha=\left(\alpha_{1}, \alpha_{2}, \ldots, \alpha_{n}\right)$ случайно равновероятно выбирается из множества $S[s]$. Нас интересует распределение случайной величины $\xi_{[s], m}=X(\alpha)$.

Для нахождения распределения $\xi_{[s], m}$ мы рассмотрим вложение процесса построения последовательности $\alpha$ в многомерную неоднородную цепь Маркова. Основная идея рассматриваемого подхода состоит в следующем. В начальный момент последовательность состоит из одного элемента, а далее каждая последовательность получается из предыдущей вставкой очередного элемента. Все размещаемые элементы предварительно упорядочены, поэтому на каждом шаге можно выразить переходные вероятности цепи через соотношения в спецификации. В работе [10] этот метод назван Finite Markov Chain Imbedding Approach, а в книге [14] можно найти его подробное изложение. Ранее указанный метод позволил получить формулы для вычисления распределений ряда комбинаторных статистик, заданных на последовательностях с фиксированным составом: числа возрастаний [11], числа отрезков убывания [13], числа пар соседних элементов последовательности, имеющих вид $(i, i+1)$ [15], вектора, состоящего из чисел возрастаний, убываний и уровней [12].

Опишем процедуру, приводящую к построению равновероятной последовательности $\alpha$ со спецификацией $[s]$, подробно. Возьмем набор Set[s] спецификации $[s]=\left[s_{1}, s_{2}, \ldots, s_{m}\right]$. Введенное выше множество $S[s]-$ это все последовательности, которые можно составить из всего набора Set[s]. Элементы множества $S[s]$ будем записывать в виде строк: $\pi=(\pi(1), \ldots, \pi(n))$. Если $[s]=\left[1^{n}\right]=[1, \ldots, 1]$, то $m=n$ и $S[s]=S_{n}$. 
Для $t=1, \ldots, n$ определим числа

$$
\widetilde{k}_{t}=\min \left\{k \in \mathbb{N} \mid \sum_{i=1}^{k} s_{i} \geqslant t\right\}, \widetilde{s}_{t}=t-\sum_{i=1}^{\widetilde{k}_{t}-1} s_{i} .
$$

Иначе говоря, если набор Set $[s]$ упорядочить по возрастанию, то на месте с номером $t$ окажется $\widetilde{S}_{t}$-й экземпляр знака $\widetilde{k}_{t}$.

Случайная последовательность $\alpha$ строится за $n$ шагов в результате размещения в одной строке знаков, выбираемых из набора Set[s] в порядке неубывания. При этом $\widetilde{S}_{t}$-й экземпляр знака $\widetilde{k}_{t}$ размещается на шаге $t$ вставкой в один ряд с $t-1$ ранее размещенными элементами на любое из $t$ мест (включая места слева и справа от всех) с вероятностью $1 / t, t=1, \ldots, n$. После шага $t$ оказываются размещенными $t$ знаков, а именно все знаки, меньшие $\widetilde{k}_{t}$, а также $\widetilde{s}_{t}$ экземпляров знака $\widetilde{k}_{t}$. Набор знаков, размещенных на шагах с первого по $t$-й, обозначим $\operatorname{Set}_{t}[s]$.

Множество всех последовательностей, которые можно составить из всего набора $\operatorname{Set}_{t}[s]$, обозначим $\Pi_{t}[s]$, а сами последовательности из $\Pi_{t}[s]$ будем записывать в виде строк: $\pi_{t}=\left(\pi_{t}(1), \ldots, \pi_{t}(t)\right)$.

Ясно, что $\Pi_{n}[s]=S[s]$. Строка $\pi_{n}$, полученная после размещения последнего $n$-го элемента, - это и есть интересующая нас последовательность $\alpha$, причем по построению все ее $n ! / \prod_{j=1}^{m} s_{j}$ ! возможных реализаций имеют равные вероятности. Таким образом, в процедуре построения последовательности $\alpha$ участвуют последовательность наборов $\left\{\operatorname{Set}_{t}[s]\right\}_{t=1}^{n}$, последовательность множеств $\left\{\Pi_{t}[s]\right\}_{t=1}^{n}$ и случайная последовательность строк $\left\{\pi_{t}\right\}_{t=1}^{n}$. Образуем случайную последовательность $\left\{X_{t}\right\}, X_{t}=X\left(\pi_{t}\right)$, где, напомним, $X\left(\pi_{t}\right)$ - число чтений строки $\pi_{t} \in \Pi_{t}[s], t=1, \ldots, n$. Заметим, что $\xi_{[s], m}=X_{n}$.

Последовательность $\left\{X_{t}\right\}$ еще не является цепью Маркова. Чтобы построить цепь Маркова, введем две вспомогательные случайные последовательности $\left\{V_{t}\right\}$ и $\left\{W_{t}\right\}$, значения которых на шаге $t$ указывают левую и правую границы расположения знаков $\widetilde{k}_{t}$ в пределах соответствующего чтения. Дадим необходимые определения.

Пусть $1 \leqslant t \leqslant n$. Рассмотрим последнее в ходе построения последовательности $\pi_{t}$ чтение, т. е. подпоследовательность $\pi_{t}\left(i_{1}\right), \ldots, \pi_{t}\left(i_{r}\right)$, элементы которой в соответствующем порядке образуют чтение последовательности $\pi_{t}$ и содержат последний размещенный элемент: $\max \left(\pi_{t}\left(i_{1}\right), \ldots, \pi_{t}\left(i_{r}\right)\right)=\widetilde{k}_{t}$. 
Положим

$$
\begin{gathered}
V\left(\pi_{1}\right)=0, W\left(\pi_{1}\right)=1, \\
\quad \begin{array}{ll}
0, & \text { если } \pi_{t}\left(i_{1}\right)=\cdots=\pi_{t}\left(i_{r}\right)=\widetilde{k}_{t}, \\
\max \left\{i_{j} \mid \pi_{t}\left(i_{j}\right)<\widetilde{k}_{t}, j=1, \ldots, r\right\} & \text { в остальных случаях, } \\
W\left(\pi_{t}\right)=\max \left\{i \mid \pi_{t}(i)=\widetilde{k}_{t}, i=1, \ldots, t\right\},
\end{array}
\end{gathered}
$$

и образуем случайные величины $V_{t}=V\left(\pi_{t}\right), W_{t}=W\left(\pi_{t}\right)$.

Событие $\left\{V_{t}=0\right\}$ состоит в том, что рассматриваемое последнее чтение перестановки $\pi_{t}$ не содержит знаков, отличных от $\widetilde{k}_{t}$. В противном случае знаки $k_{t}$ располагаются в строке $\pi_{t}$ правее места с номером $V_{t}$, а все другие знаки последнего чтения располагаются на местах с номерами не больше $V_{t}$, причем место с номером $V_{t}$ обязательно занято.

По построению набор $\left(X_{t}, V_{t}, W_{t}\right)$ принимает значения из множества

$$
\Omega_{t}=\{(x, v, w), x=1,2, \ldots, \min (t, m), 0 \leqslant v<w \leqslant t\} .
$$

Таким образом, построены отображения $T_{t}: \Pi_{t}[s] \rightarrow \Omega_{t}, t=1, \ldots, n$, вида

$$
T_{t}\left(\pi_{t}\right)=\left(X_{t}, V_{t}, W_{t}\right)=\left(X\left(\pi_{t}\right), V\left(\pi_{t}\right), W\left(\pi_{t}\right)\right)
$$

Рассмотрим евклидово пространство $\mathbb{R}^{\omega_{t}}$ размерности $\omega_{t}=\left|\Omega_{t}\right|$. Единичным координатным векторам в пространстве $\mathbb{R}^{\omega_{t}}$ можно взаимно однозначно сопоставить элементы множества $\Omega_{t}$. С учетом такого соответствия элементы $(x, v, w) \in \Omega_{t}$ можно рассматривать как векторы в пространстве $\mathbb{R}^{\omega_{t}}$. Сформулируем основной результат.

Теорема 1. Пусть для $t=1, \ldots, n$ множество $\Pi_{t}[s]$ coстоит из всех перестановок набора Set $_{t}[s]$. Тогда

(i) последовательность $\left\{T_{t}\right\}_{t=1}^{n}$ есть (неоднородная) конечная цепь Маркова на последовательности пространств состояний $\left\{\Omega_{t}\right\}_{t=1}^{n}$ с матрицами переходных вероятностей $\boldsymbol{M}_{t}=\left[P_{(x, v, w),\left(x^{\prime}, v^{\prime}, w^{\prime}\right)}(t)\right]$, где для всех пар $(x, v, w) \in \Omega_{t-1} u\left(x^{\prime}, v^{\prime}, w^{\prime}\right) \in \Omega_{t}$

$$
P_{(x, v, w),\left(x^{\prime}, v^{\prime}, w^{\prime}\right)}(t)=\mathbf{P}\left\{T_{t}=\left(x^{\prime}, v^{\prime}, w^{\prime}\right) \mid T_{t-1}=(x, v, w)\right\}=
$$




$$
= \begin{cases} & \text { либо } \quad x^{\prime}=x, v^{\prime}=w, w<w^{\prime} \leqslant t, \widetilde{s}_{t}=1, \\ \frac{1}{t}, & \text { если либо } \quad x^{\prime}=x+1, v^{\prime}=0,1 \leqslant w^{\prime} \leqslant w \leqslant t, \widetilde{s}_{t}=1, \\ & \text { либо } x^{\prime}=x, v^{\prime}=v, w+2 \leqslant w^{\prime} \leqslant t, \widetilde{s}_{t}>1, \\ \frac{w-v+1}{t}, & \text { если } \quad x^{\prime}=x, v^{\prime}=v, w^{\prime}=w+1, \widetilde{s}_{t}>1, \\ \frac{v}{t}, & \text { если } x^{\prime}=x+1, v^{\prime}=0, w^{\prime}=w+1, \widetilde{s}_{t}>1, \\ 0 & \text { в остальньх случаях; }\end{cases}
$$

(ii) распределение случайной величины $\xi_{[s], m}=X_{n}$ выражается формулой

$$
\mathbf{P}\left\{X_{n}=r\right\}=\left(\prod_{t=2}^{n} \boldsymbol{M}_{t}\right) U(r),
$$

где вектор-столбеи $U(r)$ равен сумме векторов пространства $\mathbb{R}^{\omega_{t}}$, отвечающих элементам $(r, v, w) \in \Omega_{n}, 0 \leqslant v<w \leqslant n$.

Доказательство. На первом шаге в строке единственным образом размещается самый младший элемент набора $\operatorname{Set}[s]$, который и составляет единственное чтение: $\mathbf{P}\left\{T_{1}=(1,1,1)\right\}=1$. Рассмотрим последующие шаги $t=2, \ldots, n$; пусть $T_{t-1}=(x, v, w) \in \Omega_{t-1}, T_{t}=\left(x^{\prime}, v^{\prime}, w^{\prime}\right) \in \Omega_{t}$. На шаге $t$ должен быть размещен $\widetilde{s}_{t}$-й экземпляр знака $\widetilde{k}_{t}$, причем всего существует $t$ равновероятных вариантов размещения.

Рассмотрим ситуацию, когда $\widetilde{s}_{t}=1$, т. е. среди $t-1$ ранее размещенных знаков нет знаков $\widetilde{k}_{t}$, а размещаемый знак превосходит все ранее размещенные. По определению $W_{t}$ полагается равным номеру места в строке $\pi_{t}$, занимаемого на шаге $t$. При размещении знака $\widetilde{k}_{t}$ правее места с номером $W_{t-1}$ не возникает новое чтение, а при размещении на место с номером $W_{t-1}$ или левее - возникает. Соответствующая переходная вероятность при размещении правее места с номером $W_{t-1}\left(\pi_{t-1}\right)$ равна

$$
\mathbf{P}\left\{T_{t}=(x, w, l) \mid T_{t-1}=(x, v, w)\right\}=1 / t, l=w+1, \ldots, t,
$$

а в противном случае

$$
\mathbf{P}\left\{T_{t}=(x+1,0, l) \mid T_{t-1}=(x, v, w)\right\}=1 / t, l=1, \ldots, w .
$$

Рассмотрим теперь ситуацию, когда $\widetilde{s}_{t}>1$, т. е. среди $t-1$ ранее размещенных знаков уже имеется $\widetilde{s}_{t}-1 \geq 0$ знаков $\widetilde{k}_{t}$. В этой ситуации важно, образовали ранее размещенные знаки $\widetilde{k}_{t}$ новое чтение перестановки $\pi_{t-1}$ или 
нет. Рассмотрим сначала случай, когда ранее размещенные знаки $\widetilde{k}_{t}$ не образовали нового чтения; он характеризуется условием $V_{t-1}>0$. При этом если очередной знак $\widetilde{k}_{t}$ размещается на место с номером $V_{t-1}$ или левее, то образуется новое чтение, а номер места самого правого знака $\widetilde{k}_{t}$ увеличивается на единицу. Соответствующая переходная вероятность равна

$$
\mathbf{P}\left\{T_{t}=(x+1,0, w+1) \mid T_{t-1}=(x, v, w)\right\}=v / t .
$$

Если очередной знак $\widetilde{k}_{t}$ размещается либо между местами с номерами $V_{t-1}$ и $W_{t-1}$, либо на место с номером $W_{t-1}$ или $W_{t-1}+1$, то новое чтение не образуется, а номер самого правого из размещенных знаков $\widetilde{k}_{t}$ увеличивается на единицу. Соответствующая переходная вероятность равна

$$
\mathbf{P}\left\{T_{t}=(x, v, w+1) \mid T_{t-1}=(x, v, w)\right\}=(w-v+1) / t .
$$

Если очередной знак $\widetilde{k}_{t}$ размещается правее места с номером $W_{t-1}$, но не рядом с ним, то новое чтение не образуется. При этом размещаемый знак оказывается правее всех ранее размещенных знаков $\widetilde{k}_{t}$, и номер его места присваивается величине $W_{t}$. Соответствующая переходная вероятность равна

$$
\mathbf{P}\left\{T_{t}=(x, v, l) \mid T_{t-1}=(x, v, w)\right\}=1 / t, l=w+2, \ldots, t .
$$

Рассмотрим теперь два случая, когда ранее размещенные знаки $\widetilde{k}_{t}$ уже образовали новое чтение, или, что то же самое, $V_{t-1}=0$. Если очередной знак $\widetilde{k}_{t}$ размещается на место с номером $W_{t-1}$, либо слева, либо на место с номером $W_{t-1}+1$, то новое чтение не образуется. При этом номер самого правого из ранее размещенных знаков $\widetilde{k}_{t}$ увеличивается на единицу. Соответствующая переходная вероятность равна

$$
\mathbf{P}\left\{T_{t}=(x, 0, w+1) \mid T_{t-1}=(x, 0, w)\right\}=(w+1) / t .
$$

Если очередной знак $\widetilde{k}_{t}$ размещается правее места с номером $W_{t-1}$, но не рядом с ним, то новое чтение не образуется. При этом размещаемый знак оказывается правее всех ранее размещенных знаков $\widetilde{k}_{t}$, и номер его места присваивается величине $W_{t}$. Соответствующая переходная вероятность равна

$$
\mathbf{P}\left\{T_{t}=(x, 0, l) \mid T_{t-1}=(x, 0, w)\right\}=1 / t, l=w+2, \ldots, t .
$$

Объединяя все рассмотренные случаи, получаем утверждение (i) теоремы. Утверждение (ii) следует из уравнения Колмогорова-Чепмена. 
ЗАМЕчАНИЕ 1. Доказанная теорема позволяет непосредственно выписать выражения для моментов $\mathbf{E}\left\{X_{n}^{k}\right\}$ и производящей функции $G_{X_{n}}(z)$. Положив

$$
F(k)=\sum_{r=1}^{m} r^{k} U(r) \text { и } G(z)=\sum_{r=1}^{m} z^{r} U(r),
$$

получим

$$
\mathbf{E}\left\{X_{n}^{k}\right\}=\theta_{1}\left(\prod_{t=2}^{n} \mathbf{M}_{t}\right) F(k) \text { и } G_{X_{n}}(z)=\theta_{1}\left(\prod_{t=2}^{n} \mathbf{M}_{t}\right) G(z) .
$$

ЗАМЕчАНИЕ 2. При спецификации $[s]=[1,1, \ldots, 1]$ последовательность $\alpha$ является перестановкой. В этом случае вычисления в соответствии с утверждением (i) теоремы 1 происходят аналогично вычислению чисел Эйлера по рекуррентному закону (1).

Для сравнения рассмотрим некоторые результаты, полученные ранее. В работе [2] приведены формулы для математического ожидания и дисперсии случайной величины $\xi_{[s], m}$ :

$$
\boldsymbol{E} \xi_{[s], m}=m-\sum_{r=1}^{m-1} h_{r}, \mathbf{D} \xi_{[s], m}=\sum_{r=1}^{m-1} h_{r}\left(1-h_{r}\right)+2 \sum_{k=1}^{m-2}\left(h_{r, r+1}-h_{r} h_{r+1}\right),
$$

и доказано неравенство

$$
\sup _{x}\left|\mathbf{P}\left\{\frac{\xi_{[s], m}-\mathbf{E} \xi_{[s], m}}{\sqrt{\mathbf{D} \xi_{[s], m}}} \leqslant x\right\}-\Phi(x)\right| \leqslant \frac{c H}{\left(\mathbf{D} \xi_{[s], m}\right)^{3 / 2}},
$$

где $c<\infty$ - некоторая абсолютная константа, $\Phi(x)$ - функция стандартного нормального распределения,

$$
\begin{gathered}
H=\sum_{r=1}^{m-1} h_{r}\left(1-h_{r}\right)\left(1-2 h_{r}+2 h_{r}^{2}\right), \\
h_{r}=\frac{s_{r} ! s_{r+1} !}{\left(s_{r}+s_{r+1}\right) !}, h_{r, r+1}=\frac{s_{r} ! s_{r+1} ! s_{r+2} !}{\left(s_{r}+s_{r+1}+s_{r+2}\right) !} .
\end{gathered}
$$

Отметим случай равносоставленной последовательности: $s_{1}=s_{2}=$ $=\ldots=s_{m}$. Из (2) и (3) видно, что в этом случае $H=O(m), \mathbf{D} \xi_{[s], m}=$ $=O(m)$ при $s_{1}=$ const, $m \rightarrow \infty$, и из указанного неравенства следует асимптотическая нормальность $\xi_{[s], m}$. 
В работе [1] исследована случайная величина $\zeta_{n, m}-$ число чтений в случайной последовательности, равновероятно распределенной на множестве $M^{n}$. В частности, показано, что если $m, n \rightarrow \infty$ так, что $n e^{-n / m} \rightarrow \lambda \in$ $\in[0, \infty)$, то для любого фиксированного $k=0,1,2, \ldots$

$$
\mathbf{P}\left\{m-\zeta_{n, m}=k\right\} \rightarrow \frac{\lambda^{k}}{k !} e^{-\lambda},
$$

и установлена асимптотическая нормальность $\zeta_{n, m}$ в случае, когда $m$ растет еще быстрее: $n e^{-n / m} \rightarrow \infty$. Заметим, что при конечных значениях $m$ и $n$ распределение случайной величины $\zeta_{n, m}$ выражается через числа (1) известной формулой (см. также [3, раздел 5.1.3])

$$
\mathbf{P}\left\{\zeta_{n, m}=k\right\}=\frac{A_{n, k}}{m^{n}}\left(\begin{array}{c}
m+n-k \\
n
\end{array}\right), 1 \leqslant k \leqslant n .
$$

Сопоставление распределений $\xi_{[s], m}$ и $\zeta_{n, m}$ показывает, что спецификация последовательности существенно влияет на распределение числа чтений.

ПриМЕР 1. В таблице 1 с точностью до трех значащих цифр приведены два распределения числа чтений в последовательности длины 48 над алфавитом $1,2 \ldots, 16$. В первом случае последовательность равновероятно выбирается из последовательностей спецификации $[3,3, \ldots, 3]$ (равносоставленных последовательностей), а распределение вычислено на компьютере в соответствии с теоремой 1 . Во втором случае последовательность равновероятно выбирается из всех $16^{48}$ вариантов, а распределение вычислено по формуле (4). Для иллюстрации в последней колонке приводятся асимптотические оценки вероятностей для случая равносоставленной последовательности - значения $\int_{(x-1, x]} \Phi_{(2)}(d t)$, где $x-$ число чтений, $\Phi_{(2)}(t)-$ функция нормального распределения с параметрами, вычисленными по формулам (2): математическое ожидание 61/4, дисперсия 791/1200.

ЗАМечАнИЕ 3. Мощность множества $\Omega_{n}$ равна $\omega_{n}=\min (n, m) \times$ $\times n(n+1) / 2$, что существенно меньше числа $n ! / \prod_{j=1}^{m} s_{j}$ ! различных последовательностей из $S[s]$. Вычисление распределения числа чтений при помощи теоремы 1 требует не более $O\left(\omega_{n}^{3}\right)$ операций. Для приведенного примера вычисления заняли около 0,1 секунды работы компьютера с процессором Pentium-III и тактовой частотой 1 ГГц.

ЗАмЕчАнИЕ 4. В условиях теоремы 1 значение $W_{t}$ указывает правую границу последнего на шаге $t$ чтения. Нетрудно увидеть, что наборы 
Таблица 1

\begin{tabular}{|c|c|c|c|}
\hline Число чтений & & Вероятности & \\
\hline & $\begin{array}{c}\text { для равносостав- } \\
\text { ленной последо- } \\
\text { вательности }\end{array}$ & $\begin{array}{c}\text { для равновероят- } \\
\text { ной последова- } \\
\text { тельности }\end{array}$ & $\begin{array}{c}\text { асимптотические } \\
\text { оценки }\end{array}$ \\
\hline 1 & $2.27 \cdot 10^{-49}$ & $1.94 \cdot 10^{-44}$ & \\
\hline 2 & $2.13 \cdot 10^{-35}$ & $1.30 \cdot 10^{-30}$ & \\
\hline 3 & $2.01 \cdot 10^{-27}$ & $8.34 \cdot 10^{-23}$ & \\
\hline 4 & $6.67 \cdot 10^{-22}$ & $1.76 \cdot 10^{-17}$ & \\
\hline 5 & $9.96 \cdot 10^{-18}$ & $1.58 \cdot 10^{-13}$ & \\
\hline 6 & $2.08 \cdot 10^{-14}$ & $1.85 \cdot 10^{-10}$ & \\
\hline 7 & $1.11 \cdot 10^{-11}$ & $5.10 \cdot 10^{-8}$ & \\
\hline 8 & $2.13 \cdot 10^{-9}$ & $4.64 \cdot 10^{-6}$ & \\
\hline 9 & $1.83 \cdot 10^{-7}$ & 0.000171 & \\
\hline 10 & $8.12 \cdot 10^{-6}$ & 0.00293 & \\
\hline 11 & 0.000202 & 0.0251 & $8.25 \cdot 10^{-8}$ \\
\hline 12 & 0.00298 & 0.113 & $3.11 \cdot 10^{-5}$ \\
\hline 13 & 0.0263 & 0.271 & 0.00276 \\
\hline 14 & 0.137 & 0.339 & 0.059 \\
\hline 15 & 0.384 & 0.202 & 0.317 \\
\hline 16 & 0.449 & 0.0445 & 0.443 \\
\hline
\end{tabular}

$\left(X_{t}, V_{t}, W_{t}\right)$ можно дополнить случайными величинами, которые будут соответствовать не только границе последнего на шаге $t$ чтения, но также границам всех предыдущих чтений. Выражения для переходных вероятностей соответствующей цепи Маркова будут более громоздкими, чем в теореме 1 , но состояние такой цепи Маркова на последнем шаге будет содержать более подробную информацию о чтениях перестановки $\pi_{n}$ : о знаках, которые одновременно входят в одно чтение, о взаимном расположении чтений, об их длинах и т. п.

\section{Список литературы}

1. Ватутин B.A. Предельные теоремы для числа отрезков возрастания в случайных перестановках, порождаемых алгоритмами сортировки. Дискретная математика, 1994, т. 6, вып. 1, с. 83-99.

2. Ватутин В.А., Михайлов В.Г. О числе чтений случайных неравновероятных файлов при устойчивой сортировке. - Дискретная математика, 1996, т. 8, вып. 2, с. 14-30. 
3. Кнут Д. Искусство программирования, т. 3. Сортировка и поиск. - М.: «Вильямс», 2000.

4. Риордан Дж. Введение в комбинаторный анализ. - М.: Изд-во иностранной литературы, 1963.

5. Сачков В.Н. Введение в комбинаторные методы дискретной математики. - М.: МЦНМО, 2004.

6. Эйлер Л. Дифференциальное исчисление. - М.: ГИТТЛ, 1949.

7. Carlitz L., Roselle D.P., Scoville R. Permutations and sequences with repetitions by number of increases. - J. Comb. Theory, 1966, v. 1, p. 350-374.

8. Dilon J. F., Roselle D. R. Simon Newcomb's problem. - SIAM J.Appl. Math., 1969, v. 17, № 6, p. 1086-1093.

9. Foata D. Distributions eulériennes et mahoniennes sur le groupe des permutations. - Higher combinatorics. Proc. NATO Adv. Study Inst., Berlin, 1976, ed. M. Aigner, Amsterdam, D. Reidel, 1977, p. 27-49, перевод: Фоата Д., Распределения типа Эйлера и типа Макмагона на группе перестановок. - Проблемы комбинаторного анализа, вып. 19, М.: Мир, 1980, c. $120-141$.

10. Fu J. C., Koutras M. V. Distribution theory of runs: A Markov chain approach. - J. Amer. Statist. Assoc., 1994, v. 89, p. 1050-1058.

11. Fu J.C., Lou W.Y.W. Exact and limiting distributions of the number of successions in a random permutation. - Ann. Inst. Statist. Math., 1995, v. 47, № 3, p. 435-446.

12. Fu J. C., Lou W. Y.W. Joint distributions of rises and falls. - Ann. Inst. Statist. Math., 2000, v. 52, № 3, p. 415-425.

13. Fu J. C., Lou W. Y. W., Wang Y.-J. On exact distributions of Eulerian and Simon Newcomb numbers associated witn random permutations. - Stat. \& Probab. Letters, 1999, v. 42, p. 115-125.

14. Fu J.C., Lou W.Y.W. Distribution theory of runs and patterns and its application. A finite Markov chain imbedding approach. - World Scientific Publishing Co. Pte. Ltd., Singapore, 2003.

15. Johnson C.B. The distribution of 2 -sequences in random permutations of arbitrary multi-sets. - Stat. \& Probab. Lett., 2002, v. 59, p. 67-74.

16. Rawlings $D$. Enumerations of permutations by descends, indescends, imajor index, and basic components. - J. Comb. Theory, Ser. A, 1984, v. 36, p. 1-14.

17. Wolfowitz $J$. Asymptotic distribution of runs up and down. - Ann. Math.Statist., 1944, v. 15, № 2, p. 163-172. 